\title{
Limitations of ultrasonography for diagnosing white matter damage in preterm infants
}

\author{
T Debillon, S N'Guyen, A Muet, M P Quere, F Moussaly, J C Roze
}

Arch Dis Child Fetal Neonatal Ed 2003;88:F275-F279

See end of article for authors' affiliations

.....................

Correspondence to: Dr Debillon, Service de Néonatologie, Hôpital Mère-Enfant, 9 Quai Moncousu, CHRU Nantes 44 093, Nantes Cedex 01 , France; thierry.debillon@ chu-nantes.fr

Accepted

5 September 2002

\begin{abstract}
Objectives: To compare the accuracy of ultrasonography (US) and magnetic resonance imaging (MRI) in diagnosing white matter abnormalities in preterm infants and to determine the specific indications for MRI.

Design: Prospective cohort study.

Setting: A neonatal intensive care unit in France.

Patients: All preterm infants ( $\leqslant 33$ weeks gestation) without severe respiratory distress syndrome precluding MRI.

Main outcome measures: US and MRI performed contemporaneously during the third postnatal week were analysed by an independent observer. The findings were compared with those of a term MRI scan, the results of which were taken as the final diagnosis. Statistical analysis was performed to determine which early imaging study best predicted the term MRI findings.

Results: The early US and MRI findings (79 infants) correlated closely for severe lesions (cystic periventricular leucomalacia and parenchymal infarction; $\kappa$ coefficient $=0.86$ ) but not for moderate lesions (non-cystic leucomalacia and parenchymal punctate haemorrhages; $\kappa=0.62$ ). Overall, early MRI findings predicted late MRI findings in 98\% of patients (95\% confidence interval (CI) 89.5 to 99.9) compared with only $68 \%$ for early US $(95 \% \mathrm{Cl} 52.1$ to 79.2$)$.

Conclusions: US is highly effective in detecting severe lesions of the white matter in preterm infants, but MRI seems to be necessary for the diagnosis of less severe damage. MRI performed at about the third week of life is highly predictive of the final diagnosis at term.
\end{abstract}

$\mathrm{P}$ eriventricular leucomalacia (PVL), which is characterised by multifocal zones of necrosis in the periventricular white matter, is a major cause of cerebral palsy in premature infants. However, in 1999, Paneth ${ }^{1}$ emphasised the importance of other patterns of white matter injury and suggested that the term "white matter damage" (WMD) should be used to encompass all lesions in this area, whether ischaemic or haemorrhagic, cystic or non-cystic. For both parents and doctors, predicting the outcome in a premature infant is of the utmost importance. Early diagnosis of all patterns of WMD is crucial for accurate outcome prediction.

In clinical practice, detection of WMD relies chiefly on ultrasonography (US). US changes denote development of cystic PVL in the first postnatal week. ${ }^{2}$ Acute lesions are seen as inhomogeneous echodensities which usually develop into multiple, small, echolucent cysts by the third week. The US appearance of parenchymal infarction has also been described, ${ }^{34}$ with emphasis on the distinction from cystic PVL. ${ }^{5}$ However, it has been shown that US can miss small foci of necrosis without cyst formation. ${ }^{67}$ In addition to this lack of sensitivity, there is a lack of specificity because cerebral echodensities, particularly around the frontal horns and parieto-occipital areas, are normal in premature infants. ${ }^{2}$ Moreover, the introduction of magnetic resonance imaging (MRI) has led to the identification of other patterns of WMD, mainly parenchymal punctate haemorrhages and non-cystic PVL. ${ }^{8-12}$ In a retrospective study of a cohort of preterm and term newborns, it was recently shown that MRI was more sensitive than US in detecting focal white matter lesions. ${ }^{13}$

Although MRI provides greater detail, performing serial MRI in all very low birthweight infants would raise practical difficulties not seen with US. Consequently, criteria for selecting patients for MRI need to be developed. To this end, we compared findings from contemporaneous early US and MRI in a prospective cohort of premature infants. Furthermore, these early US and MRI findings were compared with the results of term MRI. The final diagnosis was based on these late MRI findings. The objective of this study was to identify the situations in which early US accurately predicted the final diagnosis and those in which early MRI was necessary.

\section{METHODS \\ Study design}

This study was performed over one year at the neonatal intensive care unit of Nantes University Hospital. All premature infants born at or before 33 weeks gestation and alive during the third postnatal week were eligible. The only exclusion criterion was severe respiratory distress syndrome during the third postnatal week which would put the baby at undue risk during transport to the radiology unit for MRI. Informed consent was obtained from the parents.

In our unit, the regimen for US scans was an examination within the first 48 hours after birth and then once a week during the first month of life. In each study patient, two cerebral MRI scans were performed, one around 21 days of postnatal age at the same time as a cerebral US and another at term age. Four patterns of WMD were sought: parenchymal haemorrhagic infarction, parenchymal punctate haemorrhages, non-cystic PVL, and cystic PVL. Table 1 shows the criteria used to diagnose these patterns; when none were present, the MRI scan was considered normal. Cystic PVL was graded as described by de Vries et al ${ }^{14}$ : small localised cysts were defined as grade II and extensive cysts as grade III. The

Abbreviations: PVL, periventricular leucomalacia; WMD, white matter damage; US, ultrasonography; MRI, magnetic resonance imaging 
Table 1 Criteria for magnetic resonance imaging (MRI) and ultrasonography (US) diagnosis of white matter lesions

\begin{tabular}{|c|c|c|}
\hline Cerebral lesion & MRI & US \\
\hline Cystic PVL & $\begin{array}{l}\text { T1 weighted: extensive white matter lesion of signal } \\
\text { intensity similar to that of cerebrospinal fluid and } \\
\text { T2 weighted: high signal in the same area, similar to } \\
\text { the signal intensity of cerebrospinal fluid. }\end{array}$ & $\begin{array}{l}\text { Hyperechoic area (similar to the skull) with one or } \\
\text { more echo-free areas (similar to cerebrospinal fluid) } \\
\text { in the periventricular parenchyma. }\end{array}$ \\
\hline Non-cystic PVL & $\begin{array}{l}\text { T1 weighted: high signal intensity in the white matter } \\
\text { similar to the intensity of the skull and } \\
\text { T2 weighted: abnormal signal in the same area (high } \\
\text { or low according to whether haemorrhage is present). }\end{array}$ & $\begin{array}{l}\text { One or more hyperechoic areas in the periventricular } \\
\text { parenchyma. }\end{array}$ \\
\hline Parenchymal punctate haemorrhage & $\begin{array}{l}\text { T1 weighted: small circular high signal in the } \\
\text { parenchyma and } \\
\text { T2 weighted: low signal in the same area }\end{array}$ & $\begin{array}{l}\text { Small circular hyperechoic areas in the cerebral } \\
\text { parenchyma }\end{array}$ \\
\hline Parenchymal haemorrhagic infarction & $\begin{array}{l}\text { T1 weighted: unilateral, large triangular area of high } \\
\text { signal and } \\
\text { T2 weighted: low signal from the lateral ventricle and } \\
\text { adjacent parenchyma. }\end{array}$ & $\begin{array}{l}\text { Unilateral, large triangular hyperechoic area } \\
\text { involving the lateral ventricle and adjacent } \\
\text { parenchyma. }\end{array}$ \\
\hline
\end{tabular}

\begin{tabular}{lllllll} 
Table 2 & \multicolumn{7}{l}{ Pulse sequence parameters } \\
\hline $\begin{array}{l}\text { Pulse } \\
\text { sequence }\end{array}$ & $\mathrm{TR}(\mathrm{ms})$ & $\mathrm{TE} / \mathrm{T}_{\text {eff }}(\mathrm{ms})$ & $\begin{array}{l}\text { Slice } \\
\text { thickness } \\
(\mathrm{mm})\end{array}$ & $\begin{array}{l}\text { Number of } \\
\text { slices }\end{array}$ & NSA & Phase matrix \\
\hline T1-SE & 540 & 12 & 3 & 24 & 2 & 256 \\
T2-TSE & 4000 & 96 & 5 & 21 & 2 & 256 \\
\hline
\end{tabular}

SE, spin echo; TSE, turbo-spin echo; TR, repetition time; TE, echo time; $T_{\text {eff }}$ effective echo time; NSA, number of signal acquisitions.

MRI scans were read by a radiologist (MPQ) working independently from the neonatologist who performed the US scans (TD). The diagnoses based on this early MRI scan and on the contemporaneous US scan were compared. Furthermore, an independent observer (AM) in charge of prospectively collecting all the study data compared these early findings with those from a later MRI scan performed at term age. Approval for the study was obtained from the ethics committee of Nantes University Hospital.

\section{MRI and US acquisition}

MRI was performed using a 1.5 Siemens Magnetom magnetic resonance system located in the radiology unit. The magnet had a $2.54 \mathrm{~m}$ long and $0.68 \mathrm{~m}$ wide bore. Heart rate and oxygen saturation were monitored continuously, and mechanical ventilation could be continued if needed during scanning. Tl weighted spin echo and T2 weighted turbo spin echo sequences were used with the parameters shown in table 2. Cerebral US was performed using an Acusson Aspen US scanner with a transducer of $7 \mathrm{MHz}$ crystals (Acusson, Mountain View, California, USA).

\section{Statistical analysis}

Data are reported as means and ranges. Student's $t$ test was used for comparisons. $\mathrm{p}<0.05$ was considered significant. Agreement between two investigations was evaluated by calculating the $\kappa$ coefficient. As indicated by Landis and Koch, ${ }^{15} \kappa>0.81$ denotes almost perfect agreement, 0.61-0.8 substantial agreement, 0.41-0.6 moderate agreement, 0.210.4 fair agreement, and $0-0.2$ poor agreement.

\section{RESULTS}

Of the 110 potentially eligible premature infants, 31 were excluded because they had severe respiratory distress syndrome at the time the early MRI scan was scheduled. The 79

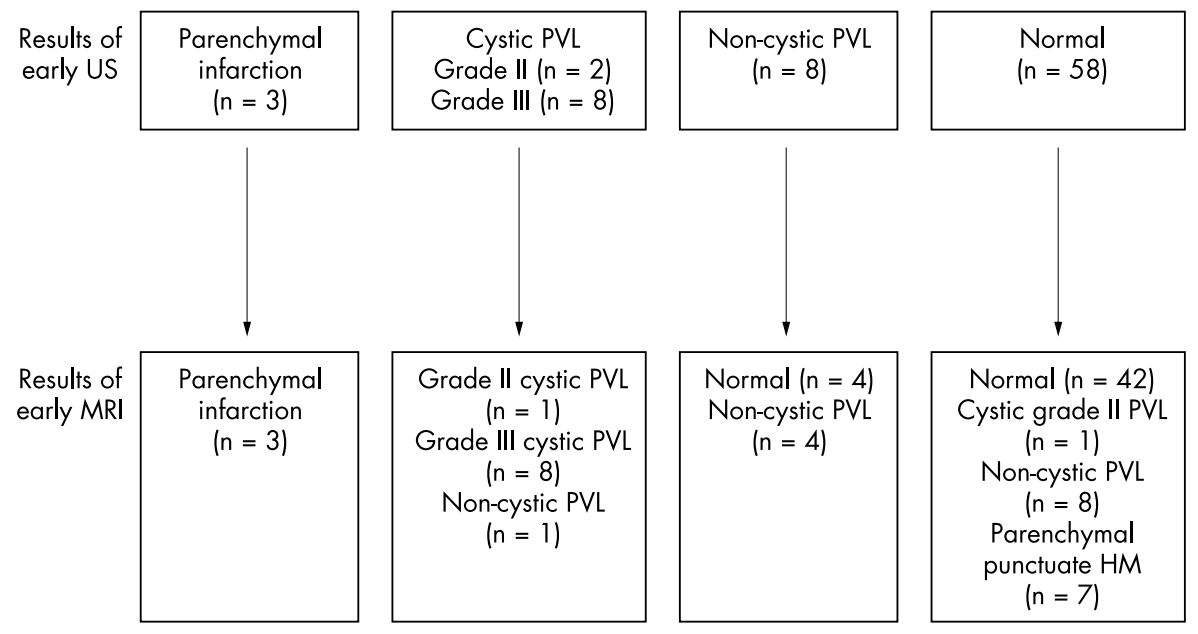

Figure 1 Comparison between the results of early ultrasonography (US) and early magnetic resonance imaging (MRI) performed on the same day in 79 preterm infants. PVL, Periventricular leucomalacia; HM, haemorrhage. 

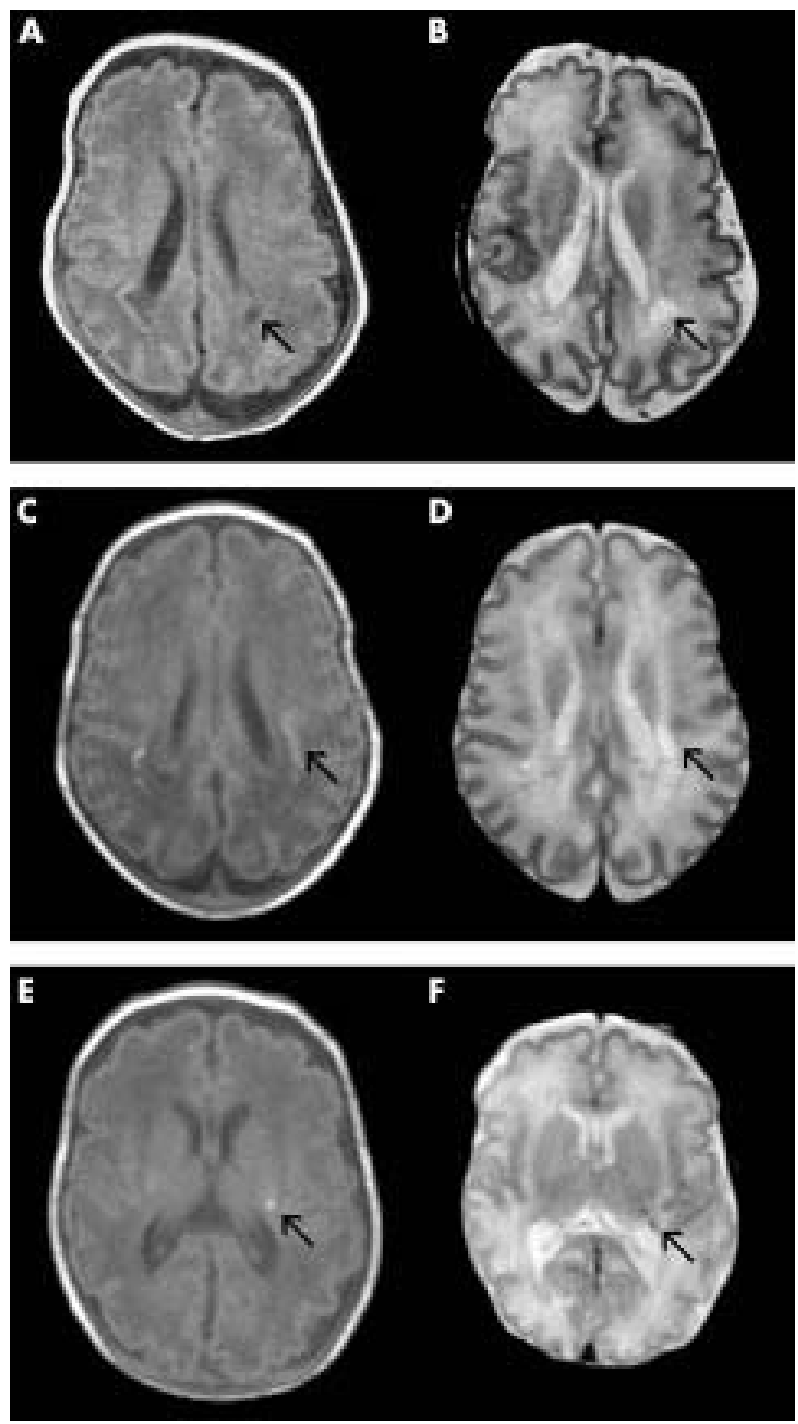

Figure 2 (A, B) Cystic periventricular leucomalacia. (A) T1 weighted image in the transverse plane low signal in the white matter (arrow) and (B) corresponding T2 weighted image in the same area showing high signal (arrow). Bilateral cysts in the white matter. (C, D) Non-cystic periventricular leucomalacia. (C) T1 weighted image in the transverse plane and (D) corresponding T2 weighted image in the same area showing high signal intensity in the white matter (arrows). (E, F) Parenchymal punctate haemorrhage. (E) Tl weighted image in the transverse plane showing circular high signal (arrow) and (F) corresponding T2 weighted image in the same area showing low signal (arrow).

infants included were born at a mean gestational age of 29.5 weeks (24-32) and had a mean birth weight of $1278 \mathrm{~g}(590-$ $2150)$. The excluded infants had a significantly younger mean gestational age $(28.7 v 29.5, \mathrm{p}<0.05)$ but a similar mean birth weight.

The early investigations (MRI and US scans) were performed at a mean postnatal age of 21 days (19-27) and at a mean postconceptional age of 31 weeks (27-36). In 51 infants, a term MRI scan was performed, at a mean postconceptional age of 37 weeks (36-47). Of the remaining 28 infants, 12 died between the early and late investigations, and 16 were withdrawn from the study because they were transferred to other neonatal intensive care units.

\section{Comparison between US and early MRI (79 infants)}

Figure 1 presents the early US and MRI findings. Overall, the $\kappa$ coefficient was 0.39 . When only the US scans showing

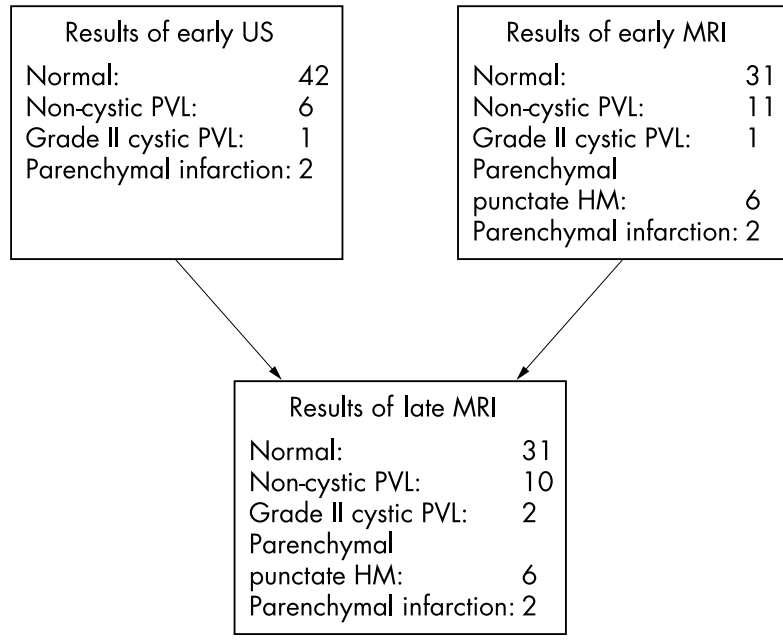

Figure 3 Comparison of the cerebral lesion diagnoses from early and late imaging in 51 preterm infants. US, Ultrasonography; MRI, magnetic resonance imaging; PVL, periventricular leucomalacia; HM, haemorrhage.

parenchymal infarction or cystic PVL (fig 2) were considered, the MRI was concordant in all the patients except one, and the $\kappa$ coefficient was 0.86 . In contrast, when US was normal or showed non-cystic PVL (fig 2), the MRI was concordant in only $69 \%$ of patients, and the $\kappa$ coefficient was 0.62 . Parenchymal punctate haemorrhage (fig 2) was seen on seven early MRI scans and on none of the US scans.

\section{Comparison between the early investigations and late MRI (51 infants)}

Figure 3 shows the term MRI findings. The early MRI predicted the results of the term MRI in 50 of 51 patients (98\%; 95\% confidence interval (CI) 89.5 to 99.9), compared with only 35 patients for US (35/51, 68\%; 95\% CI 52.1 to 79.2). The $\kappa$ coefficients were 0.96 and 0.4 respectively. The only patient whose early MRI scan failed to predict the term MRI scan results had non-cystic PVL on the early MRI and cystic MRI on the late MRI. Table 3 gives the diagnoses in the 16 patients with discordant US and term MRI findings.

\section{DISCUSSION}

In this cohort of preterm infants born at or before 33 weeks, early MRI was better than early US for predicting term WMD as assessed by MRI. Thus, early MRI predicted term MRI findings in $98 \%$ of patients, compared with only $68 \%$ for early US. Early US was insensitive for detecting non-cystic PVL and punctate haemorrhages but was sensitive for detecting more severe cerebral lesions (parenchymal infarction and grade III cystic PVL). Moreover, WMD of any pattern was common in our population: at term, $39 \%$ of the 51 infants had WMD.

Of the 79 infants who underwent early investigations, $12.5 \%$ had cystic grade II or III PVL and $16 \%$ had non-cystic PVL by MRI. The rate of cystic PVL occurrence is higher than in recent studies $(2.8 \%$ and $3.6 \%$ respectively in the studies by Pierrat et $a l^{16}$ and Bass et $a l^{17}$ ) but comparable to the rate reported by Zupan et $a l^{18}(9.2 \%)$. However, the rate in our study was not determined in the entire population of infants born at $\leqslant 33$ weeks gestation, as 31 infants were excluded. Furthermore, the above studies were conducted in larger cohorts over a period of one or more years. Differences in PVL incidences among centres has previously been reported. ${ }^{19}$ It was suggested that more information on recruitment and policies need to be reported to allow comment on these differences.

Among our patients, $7.5 \%$ had isolated parenchymal punctate haemorrhages, a rate similar to that reported recently by Maalouf $e \mathrm{al}^{8}$ (6.5\% in a cohort of 46 infants born before 30 weeks gestation). 
Table 3 Early and late magnetic resonance imaging (MRI) findings in the 16 infants whose early ultrasonography and late MRI were discordant

\begin{tabular}{lll}
\hline Early US & Early MRI & Late MRI \\
\hline Normal: $n=13$ & $\begin{array}{l}\text { Parenchymal punctate HM: } n=6 \\
\text { Noncystic PVL: } n=7\end{array}$ & $\begin{array}{l}\text { Parenchymal punctate HM: } n=6 \\
\text { Noncystic PVL: } n=7\end{array}$ \\
Noncystic PVL: $n=3$ & $\begin{array}{l}\text { Normal: } n=2 \\
\text { Noncystic PVL: } n=1\end{array}$ & $\begin{array}{l}\text { Normal: } n=2 \\
\text { Cystic grade II PVL: } n=1\end{array}$ \\
\hline
\end{tabular}

In the 51 preterm infants investigated at term, early MRI was better than early US for predicting term MRI findings. However, differences were found across the various patterns of WMD. Of the 10 cases of non-cystic PVL, all were detected by early MRI, compared with only four by US. Moreover, in two infants, US suggested non-cystic PVL, but this was not confirmed on either MRI scan. Similarly, punctate haemorrhages were not found in any of the patients by US but were present in six patients by MRI. This lack of sensitivity of US performed with a $7 \mathrm{MHz}$ transducer (as usually recommended) can be ascribed to the very small size of these lesions, especially punctate haemorrhages, and to the timing of US. Use of a $10 \mathrm{MHz}$ transducer, as suggested in several studies, ${ }^{10}{ }^{16}$ would perhaps improve the sensitivity of US by detecting smaller lesions, provided that these are not too deep. However, our data suggest that, when neonatal brain imaging is performed to detect these patterns of WMD, MRI is the best investigation.

The place of cerebral US in the evaluation of preterm infants deserves discussion. We found an excellent correlation between early US and MRI for cystic grade III PVL and parenchymal infarction. Similar results have been reported by others. In a cohort of 27 preterm infants, Maalouf et al ${ }^{8}$ observed that severe white matter echogenicity on US had a good predictive probability (0.96) for the presence of haemorrhagic parenchymal infarction on MRI. In other studies, all cystic PVL cases seen by MRI were also seen by US. ${ }^{102}$ However, MRI provided additional information on the number of cysts and/or presence of haemorrhagic lesions ${ }^{10}$ or abnormal signal in the posterior limb of the internal capsule at the term period. ${ }^{20}$ Although this additional information is probably useful for determining the nature of severe WMD, we suggest that US may be sufficient for detecting severe WMD in clinical practice.

Routine use of MRI in very low birthweight infants during the first few weeks of postnatal life seems impractical. In our cohort, $28 \%$ of the preterm infants were excluded because they had severe respiratory distress syndrome that might have put them at risk during MRI. Thus, criteria for selecting patients for MRI are needed. We suggest that MRI may be unnecessary in patients for whom US shows severe lesions such as cystic PVL or parenchymal infarction. In contrast, MRI may be useful to further investigate hyperechoic lesions without cysts or small localised cysts in the white matter or to detect lesions when US is normal. In our cohort, $21 \%$ of the infants with normal US findings had abnormalities on MRI scans. Although abundant data have been published on outcomes of preterm infants with parenchymal infarction ${ }^{521}$ and cystic PVL, ${ }^{16}{ }^{22}$ little is known about outcomes of patients with non-cystic PVL or punctate haemorrhages. Recently, a normal neurodevelopmental outcome at 2 years was reported in $7 / 8$ preterm infants with isolated punctate brain lesions. ${ }^{12}$ Thus, further studies are needed to improve the ability of clinicians to establish a prognosis in these patients.

Because MRI cannot easily be repeated at closely spaced intervals, the best age for performing it must be determined. The high concordance rate between early and late MRI findings in our study suggests that virtually all white matter lesions are present by the third postnatal week, and therefore this may be a good age for cerebral imaging studies. In a study of serial MRI in a cohort of extremely preterm infants, excessive high signal intensity was seen in the white matter at term, but was not always visible two days after birth. ${ }^{8}$ Moreover, this indicates that MRI performed within a few days of birth can miss some patterns of WMD (unless diffusion weighted imaging is used) and that MRI during the third week, as in our study, may be optimal.

In summary, we found that cerebral US is an excellent method for detecting severe WMD but can miss gliosis without cysts and punctate haemorrhages in the white matter. These less severe lesions can be detected during the third postnatal week by MRI with T1 and T2 weighted sequences. Widespread use of MRI for patients with no or minor lesions detected by US would probably lead to an increased rate of detection of WMD. Studies are needed to determine the neurodevelopmental outcomes in patients with non-cystic or punctate haemorrhagic WMD.

\section{Authors' affiliations}

T Debillon, S N'Guyen, A Muet, J C Roze, Neonatal Intensive Care Unit, University Hospital, Nantes, France

M P Quere, F Moussaly, Radiology and Magnetic Resonance Imaging Unit, University Hospital, Nantes

\section{REFERENCES}

1 Paneth N. Classifying brain damage in preterm infants. J Pediatr $1999 ; 134: 527-9$.

2 Govaert P, de Vries LS. Pathology: white matter disease. In: Govaert P, de Vries LS, eds. An atlas of neonatal brain sonography. London: Mac Keith Press, 1997:213-65

3 Rademaker KJ, Groenendaal F, Jansen GH, et al. Unilateral haemorrhagic parenchymal lesions in the preterm infant: shape, site and prognosis. Acta Paediatr 1994;83:602-8

4 Volpe JJ. Hypoxic-ischemic encephalopathy: clinical aspects. In: Volpe JJ, ed. Neurology of the newborn. Philadelphia: WB Saunders Company, 1995:314-69

5 de Vries LS, Roetlants-Van Rijn AM, Rademaker KJ, et al. Unilateral parenchymal haemorrhagic infarction in the preterm infant. Eur J Paediatr Neurol 2001;5:139-49.

6 Hope PJ, Gould SJ, Howard S, et al. Precision of ultrasound diagnosis of pathologically verified lesions in the brains of very preterm infants. Dev Med Child Neurol 1988;30:457-71

7 Carson SC, Hertzberg BS, Bowie JD, et al. Value of sonography in the diagnosis of intracranial haemorrhage and periventricular leukomalacia: a postmortem study of 35 cases. AJNR Am J Neuroradiol 1990;11:677-83.

8 Maalouf EF, Duggan PJ, Counsell SJ, et al. Comparison of findings on cranial ultrasound and magnetic resonance imaging in preterm infants. Pediatrics 2001;107:719-27.

9 Maalouf EF, Duggan PJ, Rutherford MA, et al. Magnetic resonance imaging of the brain in a cohort of extremely preterm infants. J Pediatr 1999; 135:351-7

10 Sie LTL, van der Knaap MS, van Wezel-Meijler G, et al. Early MR features of hypoxic-ischemic brain injury in neonates with periventricular densities on sonograms. ANJR Am J Neuroradiol 2000;21:852-61.

11 de Vries LS, Groenendaal F, van Haastert IC, et al. Correlation between the degree of periventricular leukomalacia diagnosed using cranial ultrasound and MRI later in infancy in children with cerebral palsy. Neuropediatrics 1993;24:263-8.

12 Cornette LG, Tanner SF, Ramenghi LA, et al. Magnetic resonance imaging of the infant brain: anatomical characteristics and clinical significance of punctate lesions. Arch Dis Child Fetal Neonatal Ed 2000;86:F171-7.

13 Childs AM, Cornette L, Ramenghi LA, et al. Magnetic resonance and cranial ultrasound characteristics of periventricular white matter abnormalities in newborn infants. Clin Radiol 2001;56:647-55 
14 de Vries LS, Eken P, Dubowitz LMS. The spectrum of leukomalacia using cranial ultrasound. Behav Brain Res 1992;49:1-6.

15 Landis J, Koch G. The measurement of observer agreement of categorical data. Biometrics 1977;33:159-74.

16 Pierrat V, Duquennoy C, van Haastert IC, et al. Ultrasound diagnosis and neurodevelopmental outcome of localised and extensive cystic periventricular leucomalacia. Arch Dis Child Fetal Neonatal Ed 2001;84:F151-6.

17 Bass WT, Jones MA, White LE, et al. Ultrasonographic differential diagnosis and neurodevelopmental outcome of cerebral white matter lesions in premature infants. J Perinatol 1999;19:330-6.

18 Zupan V, Gonzalez P, Lacaze-Masmonteil T, et al. Periventricular leukomalacia : risk factors revisited. Dev Med Child Neurol 1996;38:1061-7.
19 Baud O, Truffert P, Debillon T, et al. Enquête sur la mortalité et les complications neurologiques graves des grands prématurés dans cinq centres de niveau III. Arch Pediatr 1999;6:350.

20 Roelants-van Rijn AM, Groenendaal F, Beek FJ, et al. Parenchymal brain injury in the preterm infant: comparison of cranial ultrasound, MRI and neurodevelopmental outcome. Neuropediatrics 2001;32: 80-9.

21 Gibson JY, Massingale TW, Graves GR, et al. Relationship of cranial median shift to outcome of very-low-birth-weight infants with periventricular hemorrhagic infarction. J Neuroimaging 1994;4: 212-17.

22 Rogers B, Msall M, Owens T, et al. Cystic periventricular leukomalacia and type of cerebral palsy in preterm infants. J Pediatr 1994;125:S1-8.

\section{Archimedes}

In order to give the best care to patients and families, paediatricians need to integrate the highest quality scientific evidence with clinical expertise and the opinions of the family. Archimedes is a bimonthly section in Archives which seeks to assist practising clinicians by providing "evidence based" answers to common questions which are not at the forefront of research but are at the core of practice.

The format of Archimedes may be familiar. A description of the clinical setting is followed by a structured clinical question and a brief report of the search. The best evidence available to answer the question is provided as a summary table (which is electronically linked to more detailed appraisals). To pull the information together, a commentary follows. Finally, to make it all much more accessible, a box provides the clinical bottom line. This month the following topics have been published:

- Are routine chest $x$ ray and ECG examinations helpful in the evaluation of asymptomatic heart murmurs?

- Does intravenous mannitol improve outcome in cerebral malaria?

- Do antipyretics prevent febrile convulsions?

Previous Archimedes questions can be found in the issues of Archives published in the Fetal and Neonatal edition months since September 2001. Readers wishing to submit their own questions-with best evidence answers-are encouraged to read the Instructions for Authors at http://www.archdischild.com. 